\title{
Direct-on-Target Microdroplet Growth Assay Applications for Clinical Antimicrobial Susceptibility Testing
}

This article was published in the following Dove Press journal: Infection and Drug Resistance

\author{
Rongrong $\mathrm{Li}^{1}$ \\ Hao Tang ${ }^{2}$ \\ Huaming $\mathrm{Xu}^{2}$ \\ Yingli Ren ${ }^{3}$ \\ Shujin $\mathrm{Li}^{1}$ \\ Jilu Shen ${ }^{2}$
}

'Department of Clinical Laboratory, Hefei Hospital Affiliated to Anhui Medical University the Second People's Hospital of Hefei City, Hefei, Anhui Province, People's Republic of China; ${ }^{2}$ The Fourth Affiliated Hospital of Anhui Medical University Laboratory, Hefei, People's Republic of China; ${ }^{3}$ The Second Affiliated Hospital of Anhui Medical University Laboratory, Hefei, People's Republic of China
Correspondence: Jilu Shen

The Fourth Affiliated Hospital of Anhui Medical University Laboratory, No. 100 Huaihai Avenue, Xinzhan District, Hefei, Anhui Province, 2300I2, People's

Republic of China

Tel +86 I5I 55152963

Email 2565989451@qq.com

\begin{abstract}
Direct-on-target microdroplet growth assay is a new technique for analysing bacterial sensitivity and mechanisms of resistance. It is based on matrix-assisted laser desorption/ionization time-of-flight mass spectrometry and allows for easy and rapid testing. Here, we describe the development and procedure of the direct-on-target microdroplet growth assay and summarise the latest clinical applications.
\end{abstract}

Keywords: matrix-assisted laser desorption/ionization time-of-flight mass spectrometry, MALDI-TOF MS, direct-on-target microdroplet growth assay, DOT-MGA, antimicrobial susceptibility testing

Matrix-assisted laser desorption/ionization time-of-flight mass spectrometry (MALDITOF MS) was a newly developed laboratory method for the identification of microorganisms in the clinic. More recently, researchers have focused on the application of MALDI-TOF MS in the detection of microbial resistance. MALDI-TOF MS detected drug sensitivity in microorganisms by measuring the enzyme activity and analyses drug resistance using a self-built library and the characteristics of the mass spectrum peak as well as the composite correlation index (CCI). ${ }^{1-3}$ Recently, researchers published a method for analysing bacterial sensitivity by direct-on-target microdroplet growth assay (DOT-MGA), ${ }^{4,5}$ which was simple, practical, and could be rapidly executed. ${ }^{6}$ It could be used to provide valuable information on potential resistance mechanisms.

The principle of this methodology was as follows: to perform DOT-MGA, microorganisms were incubated with and without the index antibiotic in nutrient broth as microdroplets directly on the target spots of MALDI-TOF MS. To avoid droplet evaporation, the target plate was cultured inside a plastic container and water was added to the bottom. At the end of the culture period, the broth was separated from the microbial cells by direct absorption of the microdroplets using absorptive material. The microbial growth in each target plate was analysed to validate whether MALDI-TOF MS successfully identified each microbial spot at different antibiotic concentrations. Since the exact concentration of antibiotics in each droplet was known, the minimum inhibitory concentration (MIC) could be calculated and microorganisms could be identified as sensitive or insensitive to certain antibiotics. ${ }^{6,7}$ By evaluating the growth in the presence of different antibiotics, it was possible to not only determine the sensitivity of an isolate but also analyse the potential mechanisms of drug resistance. 
In the initial experiments, DOT-MGA was mainly performed on gram-negative bacteria such as Enterobacteriaceae. In the study of Idelevich et al, 24 strains of Klebsiella pneumoniae and 24 strains of Pseudomonas aeruginosa were co-cultured with meropenem to rapidly detect carbapenemase resistance. The authors used microdroplets of $2,4,6,8$, and $10 \mu \mathrm{L}$ and incubated the samples for $3,4,5$, and 18 hours and found that $6 \mu \mathrm{L}$ microdroplets combined with an incubation time of 4 hours resulted in the best validity $(100 \%)$, sensitivity (100\% and $83.3 \%$ ), and specificity $(100 \%)$ for K. pneumoniae and P. aeruginosa, respectively. ${ }^{6}$ In 2020 , Shen et al extended the application of this technique to imipenem, meropenem, and ertapenem. ${ }^{8}$ The DOT-MGA outcomes in K. pneumoniae were consistent with that of the broth microdilution method and showed superior performance in Escherichia coli for ertapenem and imipenem. Moreover, Horseman et al used the same method to analyse methicillinresistant Staphylococcus aureus (MRSA), vancomycinresistant Enterococcus, extended-spectrum $\beta$-lactamase (ESBL) E. coli, and carbapenem-resistant K. pneumoniae. ${ }^{9}$ The authors demonstrated that while a droplet size of 4,6 , or $8 \mu \mathrm{L}$ did not affect the identification and detection of drug resistance, larger than $6 \mu \mathrm{L}$ droplets resulted in frequent overflow and difficulties in completely separating the broth from the microorganism. Therefore, $4 \mu \mathrm{L}$ was determined to be optimal. Furthermore, the assay showed higher validity in determining drug resistance in $S$. aureus and K. pneumoniae (97.5\%) than in E. coli (82.5\%) and Enterococcus (65\%). These data were consistent with those using the broth extraction method.

In the clinic, sensitivity analyses of microorganisms present in a patient's bloodstream were critical for determining treatment strategy. Nix et al examined DOT-MGA for the rapid detection of microbial resistance in patients using positive blood culture bottles and confirmed its applicability in MRSA using cefoxitin and determined an optimal microdroplet size of $6 \mu \mathrm{L} .{ }^{10}$ By investigating the effects of three pretreatment methods including continuous dilution broth, lysis/ centrifugation, and differential centrifugation, the authors found that lysis/centrifugation combined with an incubation time for 4 hours results in the best validity $(96.4 \%)$, sensitivity $(100 \%)$, and specificity $(100 \%)$. Importantly, it was necessary to destroy the MRSA cell membrane with formic acid before adding the matrix in contrast to performing DOT-MGA on gram-negative bacteria. Similar analyses were executed by Idelevich et al in $K$. pneumoniae, Enterobacter cloacae, Enterobacter aerogenes, Proteus mirabilis, and Klebsiella aerogenes for determining carbapenemase resistance. ${ }^{11}$ In addition to the three pre-treatment methods mentioned above, the authors also examined a filtration/dilution method and observed that lysis/centrifugation and an incubation time of 4 hours showed the best performance with a validity, sensitivity, and specificity of $96.3 \%, 91.7 \%$, and $100 \%$, respectively.

In addition to its applicability as a rapid detection method of bacterial resistance in the clinic, DOT-MGA could also be used to explore the mechanism of bacterial resistance and synergistic inhibition by phenotypic screening. In the study of Correa-Martínez et al, Enterobacteriaceae were exposed to cephalosporins and inhibitors in the microdroplets at 12 different concentrations to examine the following eight conditions: bacteria with (1) cefpodoxime, (2) ceftazidime, (3) ceftazidime/ clavulanic acid, (4) cefotaxime, (5) cefotaxime/clavulanic acid, (6) cefepime, (7) cefepime/clavulanic acid, and (8) cefotaxime/clavulanic acid/cloxacillin. ${ }^{12}$ The authors reported an 8-fold decrease in MIC when clavulanic acid and cloxacillin were added, indicating a synergistic inhibition effect and analysing potential resistance mechanisms. The standards and algorithms were proposed by EUCAST. ${ }^{13}$ Compared with PCR analyses, the positive and negative coincidence rates of ESBL, AmpC, and ESBL + AmpC were $94.4 \%$ and $100 \%, 94.4 \%$ and $93.8 \%, 100 \%$ and $100 \%$, respectively. Additionally, the authors also screened carbapenemase-resistant strains by co-culture with meropenem, in which the microorganisms were exposed to meropenem mixed with a variety of antibiotics in the microdroplets. In these experiments, DOT-MGA showed comparable results with PCR, broth dilution, and disk diffusion for KPC, MBL, and OXA. The results of AmpC were consistent with those of broth dilution and disk diffusion, although there were discrepancies with the results obtained by PCR. ${ }^{14}$ It was not ruled out that PCR results were false negative.

MALDI-TOF MS-based DOT-MGA is a promising method for identifying microorganisms as well as for analysing drug sensitivity and mechanisms of microbial resistance. It is simple to execute with rapid and accurate results and can be used to analyse both gram-positive and negative microorganisms. Furthermore, it can be directly applied to culture plates and positive blood culture bottles, which accelerates the antibiotic resistance analyses and allows for faster intervention by clinicians in patients with bacteraemia. However, it is worth noting that the standardisation of this method still needs further optimization. 


\section{Code Availability (Software Application or Custom Code)}

The authors declare the software application and custom code support their published claims and comply with field standards.

\section{Data Sharing Statement}

The authors declare that all data and materials support their published claims and comply with field standards.

\section{Author Contributions}

All authors made substantial contributions to conception and design, acquisition of data, or analysis and interpretation of data; took part in drafting the article or revising it critically for important intellectual content; agreed to submit to the current journal; gave final approval of the version to be published; and agree to be accountable for all aspects of the work.

\section{Funding}

This study was funded by The University Synergy Innovation Program of Anhui Province (GXXT-2020-016) and Research Fund of Anhui Institute of Translational Medicine (Immunoprotection of His-A12 (1-85) recombinant protein of Pneumocystis carinii) (grant number 2017zhyx14).

\section{Disclosure}

The authors declare that they have no conflict of interest.

\section{References}

1. Paul S, Singh P, Shamanth AS, Rudramurthy SM, Chakrabarti A, Ghosh AK. Rapid detection of fluconazole resistance in Candida tropicalis by MALDI-TOF MS. Med Mycol. 2018;56(2):234-241. doi:10.1093/mmy/myx042

2. Oviaño M, Rodríguez-Martínez JM, Pascual Á, Bou G, Pascual Á, Bou G. Rapid detection of the plasmid-mediated quinolone resistance determinant AAC $\left(6^{\prime}\right)$-Ib-cr in Enterobacteriaceae by MALDI-TOF MS analysis. $J$ Antimicrob Chemother. 2017;72(4):1074-1080. doi:10.1093/jac/dkw552

3. Oviaño M, Bou G. Matrix-assisted laser desorption ionization-time of flight mass spectrometry for the rapid detection of antimicrobial resistance mechanisms and beyond. Clin Microbiol Rev. 2018;32(1): e00037-18. doi:10.1128/CMR.00037-18

Infection and Drug Resistance

\section{Publish your work in this journal}

Infection and Drug Resistance is an international, peer-reviewed openaccess journal that focuses on the optimal treatment of infection (bacterial, fungal and viral) and the development and institution of preventive strategies to minimize the development and spread of resistance. The journal is specifically concerned with the epidemiology of
4. Neonakis IK, Spandidos DA. MALDI-TOF MS-based direct-ontarget microdroplet growth assay: latest developments. Exp Ther Med. 2020;20(3):2555-2556. doi:10.3892/etm.2020.8976

5. Neonakis IK, Spandidos DA. MALDI-TOF mass spectrometry-based direct-on-target microdroplet growth assay: a novel assay for susceptibility testing and beyond. Future Microbiol. 2019;14(9):729-731. doi:10.2217/fmb-2019-0106

6. Idelevich EA, Sparbier K, Kostrzewa M, Becker K. Rapid detection of antibiotic resistance by MALDI-TOF mass spectrometry using a novel direct-on-target microdroplet growth assay. Clin Microbiol Infect. 2018;24(7):738-743. doi:10.1016/j.cmi.2017.10.016

7. Neonakis IK, Spandidos DA. Detection of carbapenemase producers by matrix-assisted laser desorption-ionization time-of-flight mass spectrometry (MALDI-TOF MS). Eur J Clin Microbiol Infect Dis. 2019;38(10):1795-1801. doi:10.1007/s10096-019-03620-0

8. Shen J, Huang S, Zhou C, Bijie H, Guo W. Rapid differential diagnosis value of MALDI-TOF MS direct target plate microdrop growth method for carbapenem resistant Enterobacteriaceae. Chin Clin Med. 2020;27(04):549-553.

9. Horseman TS, Lustik MB, Fong KSK. Rapid qualitative antibiotic resistance characterization using VITEK MS. Diagn Microbiol Infect Dis. 2020;97(4):115093. doi:10.1016/j.diagmicrobio.2020.115093

10. Nix ID, Idelevich EA, Storck LM, et al. Detection of methicillin resistance in Staphylococcus aureus from agar cultures and directly from positive blood cultures using MALDI-TOF mass spectrometry-based direct-on-target microdroplet growth assay. Front Microbiol. 2020;11:232. doi:10.3389/fmicb.2020.00232

11. Idelevich EA, Storck LM, Sparbier K, Drews O, Kostrzewa M, Becker K. Rapid direct susceptibility testing from positive blood cultures by the matrix-assisted laser desorption ionization-time of flight mass spectrometry-based direct-on-target microdroplet growth assay. J Clin Microbiol. 2018;56(10):e00913-18. doi:10.1128/ JCM.00913-18

12. Correa-Martínez CL, Idelevich EA, Sparbier K, Kostrzewa M, Becker K. Rapid detection of extended-spectrum $\beta$-lactamases (ESBL) and AmpC $\beta$-lactamases in Enterobacterales: development of a screening panel using the MALDI-TOF MS-based direct-ontarget microdroplet growth assay. Front Microbiol. 2019;10:13. doi:10.3389/fmicb.2019.00013

13. EUCAST. Guidelines for detection of resistance mechanisms and specific resistances of clinical and/or epidemiological importance. The European Committee on Antimicrobial Susceptibility Testing. Version 2.0; 2017. Available from: https://www.eucast.org/resis tance_mechanisms/. Accessed April 8, 2021.

14. Correa-Martínez CL, Idelevich EA, Sparbier K, Kuczius T, Kostrzewa M, Becker K. Development of a MALDI-TOF MS-based screening panel for accelerated differential detection of carbapenemases in Enterobacterales using the direct-on-target microdroplet growth assay. Sci Rep. 2020;10(1):4988. doi:10.1038/s41598020-61890-7 antibiotic resistance and the mechanisms of resistance development and diffusion in both hospitals and the community. The manuscript management system is completely online and includes a very quick and fair peerreview system, which is all easy to use. Visit http://www.dovepress.com/ testimonials.php to read real quotes from published authors. 